\title{
Remote Management and Maintenance System Based on LPC2292 for the Vehicles
}

\author{
Z.-Q. Li \\ The University of Armed Police Engineering, Xi'an, Shanxi, China
}

\begin{abstract}
In this thesis we proposed a solution of the remote management and monitoring system for the vehicles, accounting the actual demands of the CAPF. This system is based on the advanced technology of domestic and foreign vehicle monitoring systems. The fault collection terminal is an embedded system based on LPC2292 and ECOS; the remote diagnosis server consists of the communication module, database server and the WEB server. This system integrates the functions of the remote real-time state monitoring, fault diagnosis and analysis, fault warning and daily management for the vehicles. It transforms the maintenance mode from the traditional passive repair way to a kind of active preventive way; from the timing or mileage maintenance to real-time maintenance; from the extensive management way to the fine management with great military benefits.
\end{abstract}

Keywords-Vehicles, GPRS, CAN-Bus, remote maintenance

\section{基于 LPC2292 车辆远程监控与管理系统设计}

\author{
李宗强 \\ 西安武警工程大学, 西安, 陕西, 中国
}

摘 要 本文基于国内外车辆监控的先进技术, 立足实际需求, 设计了警用车辆远程监控与管理系统的实现方案; 基于 LPC 2292 ARM7 嵌入式处理器与 ECOS 嵌入式系统, 设计开发了车载故障信息采集终端; 结合远程通讯模块、数据库服务器与 WEB 服务器于 一体, 实现了远程故障诊断服务器。该系统集警用车辆远程实时状态监测、故障诊断分析、预警和车辆日常正规化管理等多项功能。 改变车辆传统的维护模式, 由被动修复性维护转变为主动预防性维护; 将汽车的定时或定里程维护转化为实时维护; 变粗放式管理为 精细化正规化管理。

关键词 车辆, GPRS, CAN-Bus, 远程维护, 故障诊断

1. 引言

日常汽车的维护和保养随着汽车电子化程度日益提 高, 变得更加复杂。而对于绝大多数汽车驾驶员而言, 采 用过去传统的经验诊断方法来进行汽车自身的维护变得越 来越困难, 越来越需要依赖专业人员凭借专业设备与工具 的专业支持, 如何提高汽车维护的科学性与经济性, 成为 当前车辆管理的热点和难点。

因此, 我们依据实际需求, 改变车辆传统维护模式, 由被动修复性维护转变为主动预防性维护, 变粗放式管理 为精细化正规化管理, 提出一种汽车远程诊断与维护系统, 将汽车的定时 (定里程) 维护转化为实时维护, 将很大程 度地改变当前的汽车维护模式, 真正起到一种预防维护的 效果。
车辆远程监控与管理系统集车辆远程实时状态监测、 故障诊断分析、预警和警用车辆日常正规化管理等多项功 能, 通过对车辆各运行参数实时监测, 获取车内各种运行参 数和故障码, 并进行信息预判断, 一旦发现存在着故障状 态或故障隐患, 即将故障信息连同实时运行参数发出。指 挥中心能够根据反馈的信息, 评价警车的运行状态, 建议 其是否需要维护或修理等 $[1-3]$ 。该系统的使用可大大降低 车辆事故率, 降低和节约日常汽车维护成本, 确保车辆全 程监控与安全预警, 具有一定的军事效益和经济效益。

\section{2. 系统总体结构}

为降低日常车辆的维护成本, 提高日常行车的安全性, 本文将车辆的定时或定里程维护转化为实时维护。系统由 
故障信息采集终端与远程维护服务中心构成, 采集终端是 一个基于 LPC2292 与 ECOS 的嵌入式系统, 而维护服务器中 心则合通讯服务器、数据库服务器与 WEB 服务器于一体。 嵌入式汽车远程诊断与维护系统框图如图 2.1 所示。

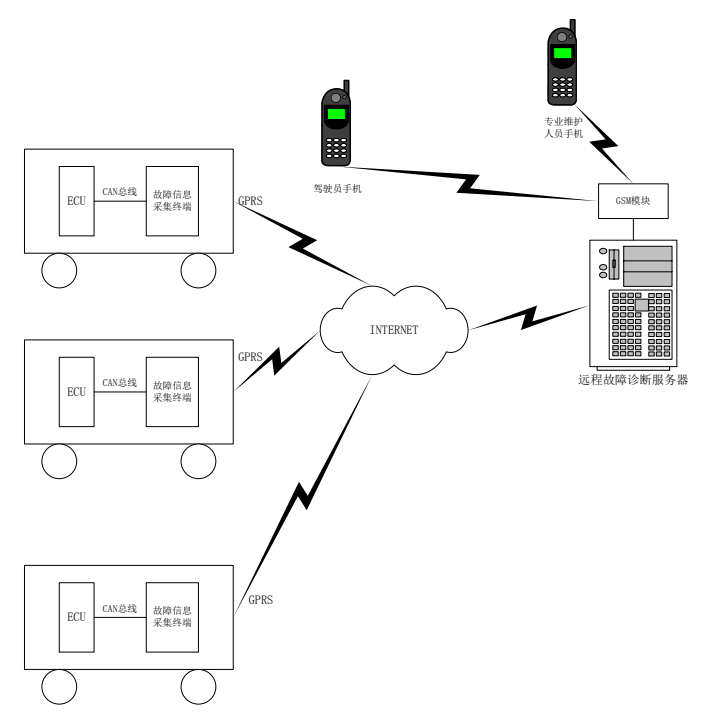

图 2.1 嵌入式汽车远程诊断与维护系统框图

主要包括二个部分：故障信息采集终端和远程故障诊 断服务器。

故障信息采集终端采用典型的嵌入式系统, 。通过 CAN 总线与 ECU 相连接, 获取汽车故障信息; 遵循 SAE J1939 协议将从 ECU 获取到的汽车故障信息进行解析, 并转化成 统一格式的故障信息列表 ${ }^{[4][5]}$; 将故障信息列表按优先级通 过 GPRS 模块进行远程发送, 通过 INTERNET 传送到安装在 服务中心的远程故障诊断服务器。

远程故障诊断服务器实时接入多个网络连接, 实现来 自于各个采集终端的数据实时接收; 建立所有需要服务的 汽车的历史维护档案 (类似于电子病历); 结合汽车的历史 维护档案, 实现专家级的汽车故障诊断, 并提出相应的维 护方案等功能。

\section{3. 故障信息采集终端硬件设计}

故障信息采集终端的任务是不停地实时读取汽车 ECU 的故障信息, 加以预判断分析, 并把分析后的数据通过 GPRS 传给远程故障诊断与维护服务器。整个终端设计采用了 “盲 终端” 方式, 安装在汽车上驾驶员不易接触的地方, 无需 单独的人机交互界面。采集终端的模块框图如图 3.1 所示。

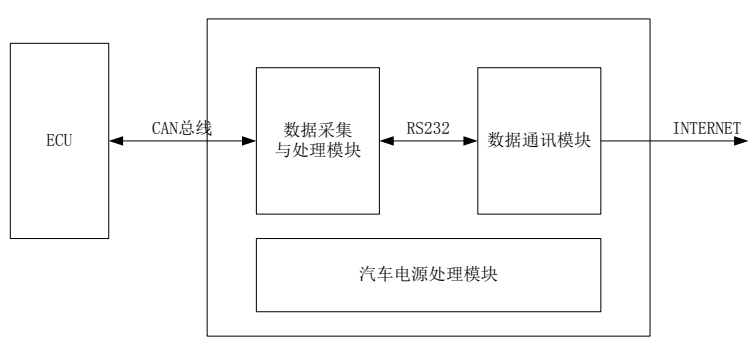

图 3.1 采集终端的模块框图

本系统的采集终端的一个任务是实时地通过 CAN 总线 与上述的 $\mathrm{ECU}$ 进行数据通讯。从传感器中采集到的原始数 据经 $\mathrm{ECU}$ 初步封装后通过 CAN 总线打包, 打包后的数据以 列表方式存入到原始数据池; 终端另一任务是自动扫描原 始数据池列表, 并根据 SAEJ1939 协议进行解析与预判断, 一旦发现有故障信息, 将根据故障状态编上优先级, 并依 次存入到处理后数据池; 另外, 终端还有一任务, 是自动 扫描处理后数据池列表, 并将列表数据打成数据包后通过 数据通讯模块传输到远程服务器。

\section{1 车载故障信息采集终端硬件系统结构}

系统的硬件框图如图 3.2 所示。数据采集与处理模块 的处理器采用了 NXP 的 ARM7 处理器 LPC2292, 其内部具有 双路带有先进的验收滤波器的 CAN 总线控制器, 并通过扩 展 TJA1040 作为 CAN 总线收发器, 实现与 ECU 的数据实时 通讯。数据通讯模块采用了索爱公司的 GR64 专用 GPRS 无 线通讯模块, 内部封装了完整的 GPRS 通讯机制与 TCP/IP 协议 ${ }^{[6]}$ 。LPC2292 处理器通过 RS232 串行接口与 GP64 模块 实现通讯。

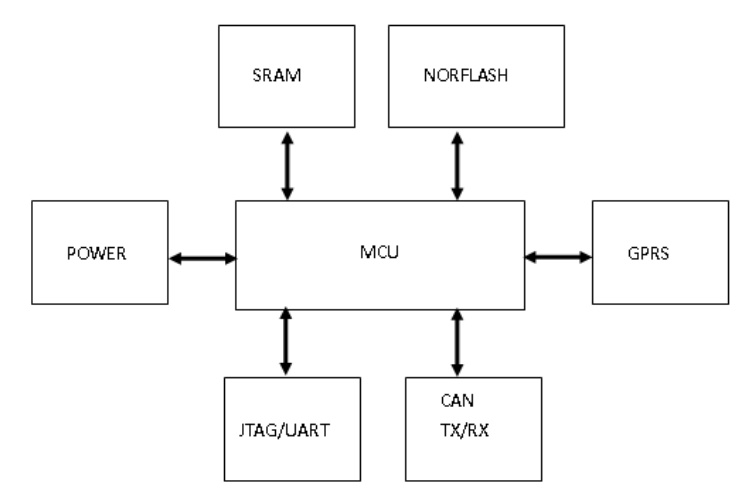

图 3.2 系统的硬件框图 


\subsection{MCU 模块硬件设计}

LPC2292 是一款基于 16/32 位 ARM7TDMI-S, 并支持实 时仿真和跟踪的 CPU, 并带有 $256 \mathrm{k}$ 字节 $(\mathrm{kB})$ 嵌入的高速 Flash 存储器。128 位 宽度的存储器接口和独特的加速结 构使 32 位代码能够在最大时钟速率下运行。对代码规模有 严格控制的应用可使用 16 位 Thumb 模式将代码 规模降低 超过 $30 \%$, 而性能的损失却很小。

\section{3 CAN 总线模块硬件设计}

LPC2292 芯片作为工业级的控制芯片, 其内部集成有 两路 CAN 控制器 ${ }^{[7]}$ 。因此系统 CAN 模块的硬件设计主要针 对 CAN 收发器部分与 CAN 控制器之间的硬件连接。

系统选用 TJA1040 作为 CAN 收发器, 其周边电路设计 如图 3.3 所示:

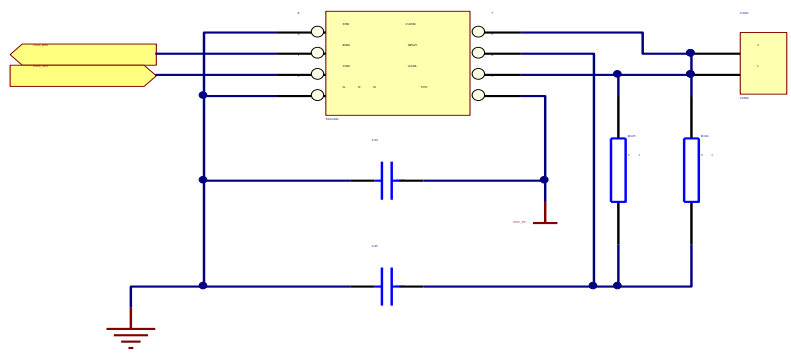

图 $3.3 \mathrm{CAN}$ 总线模块硬件接口设计

图中 CAN_RD1、CAN_TD1 分别连接 LPC2292 芯片对应管 脚, 即 CAN 控制器的对应引脚; CAN_H、CAN_L 为模块连接 网络的通讯线。

3.4 车载故障信息采集终端底层 $\mathrm{eCos}$ 操作系统移植及驱动 程序设计

考虑到汽车电子产品对实时性、可靠性的要求, 本系 统选用 eCos 嵌入式操作系统对系统资源及应用线程进行 管理。同时, 遵照 eCos 操作系统对于设备驱动的编写规范 设计本系统的设备驱动。

系统涉及到较多的设备驱动程序, 包括 Flash 驱动、 CAN 总线驱动、 $\mathrm{I} 2 \mathrm{C}$ 总线驱动、SPI 总线驱动及对普通 $\mathrm{I} / 0$ 的操作等。

\section{4. 远程故障诊断软件系统设计}

4. 1 客户端应用层程序设计

4.1.1 系统用例图

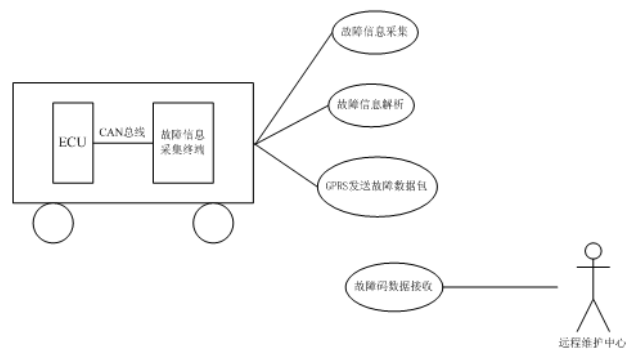

图 4.1 远程故障诊断系统客户端用例图

\subsection{2 嵌入式软件设计}

系统应用程序创建 6 个相互独立的线程, 用于实现各 自功能, 这些线程分别是: 行车实时参数信息获取线程、 开关量采集线程、实时信息处理及显示线程、故障信息处 理及 GPRS 无线网络线程、行车信息存储及按键扫描线程。

\section{2 远程故障诊断服务中心设计}

远程故障诊断服务器安装在汽车远程服务中心, 采用 前后台结构是警用车辆远程监控与管理系统的重要组成部 分。

本文针对车辆管理和维护现状, 立足车辆正规化管理 的迫切需求, 改变任务车辆传统的定时、定里程维护模式, 变被动修复性维护为主动预防性维护, 大大降低了车辆的 事故率和维护成本。

\section{参考文献(References)}

[1] Han Yu-min. Research car remote diagnosis and maintenance of the system. The use of car, 2007, (7): 30-31.

[2] Huang Da-chao, Sun De-lin. Modern automotive remote diagnostic system. Techniques of Automation and Applications, 2007.26(7): 129-130.

[3] Xu De-zhang. Modern car dashboard technology and development trends. Auto Electric, 2002, (5):1-4.

[4] Leen Gabriel1, Heffernan Donal1. Expanding automotive electronic systems. Computer. Jan 2002:88-93. 
[5] Zhou Hong, He Pei-qi, Gao Peng. Car remote maintenance system in transit. Electrical and Mechanical Engineering, 2009, (1):51-53.

[6] Chen Xin, Hu Xiao-long. Remote diagnostics automotive fault diagnostic GPRS-based design. Industrial control compute, 2006,
19(4): 70-72.

[7] Liu Lun-hong, Guo Xue-xun, Fu Chang. Information Systems Research of automotive based on CAN and LIN-bus. Wuhan University of Technology, 2008, 30(3):415-419. 